\title{
Acutely Altered Mental Status as the Main Clinical Presentation of Multiple Strokes in Critically III Patients With COVID-19.
}

Carolina Díaz-Pérez ( $\nabla$ carodipez@gmail.com )

La Princesa University Hospital https://orcid.org/0000-0003-3661-5368

\section{Carmen Ramos}

La Princesa University Hospital

Alberto López-Cruz

La Princesa University Hospital

José Muñoz Olmedo

La Princesa University Hospital

Jimena Lázaro González

La Princesa University Hospital

\section{Enrique de Vega-Ríos}

La Princesa University Hospital

\section{Carmen González-Ávila}

La Princesa University Hospital

\section{Carlos Hervás}

La Princesa University Hospital

\section{Santiago Trillo}

La Princesa University Hospital José Vivancos

La Princesa University Hospital

\section{Short Report}

Keywords: COVID-19, acutely altered mental status, multiple strokes

Posted Date: May 29th, 2020

DOI: https://doi.org/10.21203/rs.3.rs-31769/v1

License: (1) (1) This work is licensed under a Creative Commons Attribution 4.0 International License. Read Full License 
Version of Record: A version of this preprint was published at Neurological Sciences on August 17th, 2020. See the published version at https://doi.org/10.1007/s10072-020-04679-w. 


\section{Abstract}

\section{Background and aims:}

Cerebral infarction in COVID-19 patients might be associated with a hypercoagulable state related to a systemic inflammatory response. Its diagnosis might be challenging. We present two critically ill patients with COVID-19 who presented acutely altered mental status as the main manifestation of multiple strokes.

\section{Methods:}

Clinical presentation and diagnostic work-up of the patients.

\section{Results:}

Two patients in their sixties were hospitalized with a bilateral pneumonia COVID-19. They developed respiratory failure and were admitted to ICU for mechanical ventilation and intense medical treatment. They were started on low-molecular-weight heparin since admission. Their laboratory results showed lymphopenia and increased levels of C-reactive protein and D-dimer. Case 1 developed hypofibrinogenemia and presented several cutaneous lesions with biopsy features of thrombotic vasculopathy. Case 2 was performed a CT pulmonary angiogram at ICU showing a bilateral pulmonary embolism. When waking up, both patients were conscious but with a remarkable global altered mental status without focal neurological deficits. A brain MRI revealed multiple acute bilateral ischemic lesions with areas of hemorrhagic transformation in both patients (Case 1: affecting the left frontal and temporal lobes and both occipital lobes; Case 2: affecting both frontal and left occipital lobes). Cardioembolic source and acquired antiphospholipid syndrome were ruled out. COVID-19-associated coagulopathy was suspected as the possible main etiology of the strokes.

\section{Conclusion:}

Acutely altered mental status might be the main manifestation of multiple brain infarctions in critically ill COVID-19 patients. It should be specially considered in those with suspected COVID-19-associated coagulopathy. Full-dose anticoagulation and clinical-radiological monitoring might reduce their neurological consequences.

\section{Introduction}

The COVID-19 caused by the novel coronavirus SARS-CoV-2 usually manifests with fever, cough and dyspnea. Its characteristic radiological findings are bilateral areas of ground-glass opacity on chest CT images. Some of these patients progress to an inflammatory phase which can be life threatening, requiring mechanical ventilation (MV) and admission to the intensive care unit (ICU) [1]. 
In severe forms of the disease, several neurological manifestations have been described, mostly affecting the central nervous system [2]. One of these neurological complications is cerebral infarction, which may be associated with a hypercoagulable state with micro- and macro-circulatory thrombosis in the context of a systemic inflammatory response [3].

It is not unusual for critically ill patients to develop brain ischemic lesions whose symptoms are not identified as a clearly focal neurological deficit. We present two critically ill patients with COVID-19 who presented acutely altered mental status as the main manifestation of multiple strokes.

\section{Results}

\section{CASE 1}

A 61-years-old-man presented with a bilateral pneumonia COVID-19 on the 3rd day of symptoms. He was admitted to the internal medicine service (IMS) on hydroxychloroquine, lopinavir/ritonavir and lowmolecular-weight heparin (LMWH) $60 \mathrm{mg}$ q.d.

After two days he developed respiratory failure. Laboratory results showed lymphopenia (300 cells/mcl) and increased levels of C-reactive protein $(32.2 \mathrm{mg} / \mathrm{dl})$, ferritin $(538 \mathrm{ng} / \mathrm{ml})$, fibrinogen $(631 \mathrm{mg} / \mathrm{dl})$ and Ddimer $(124.86 \mu \mathrm{g} / \mathrm{ml})$. He was admitted to the ICU for MV, receiving systemic corticosteroids and two doses of tocilizumab. A remarkable complication was hypofibrinogenemia ( $73 \mathrm{mg} / \mathrm{dl})$, solved after fibrinogen and vitamin $\mathrm{K}$ supplementation.

After clinical and analytical improvement he was discharged back to the IMS. Neurological examination revealed drowsiness, inattention, disorientation, poverty of content of speech and increased latency of response to orders with no focal neurologic deficits. The symptoms fluctuated and psychomotor agitation partially responded to quetiapine. The electroencephalogram pattern was suggestive of moderate diffuse encephalopathy.

A brain MRI was performed which demonstrated multiple ischemic lesions visible on DWI and FLAIR images affecting the left frontal and temporal lobes and both occipital lobes with extensive areas of hemorrhagic transformation on SWI sequence (Fig. 1 a, b, c).

Cardioembolic source was ruled out and acquired antiphospholipid syndrome study was normal. He also developed several cutaneous lesions whose biopsy showed features of thrombotic vasculopathy, being a COVID-19-associated coagulopathy the main etiology suspected for the ischemic lesions.

\section{CASE 2}

A 65-years-old man with hypertension and diabetes mellitus presented with a bilateral pneumonia COVID19 on his 7th day of symptoms, with mild respiratory compromise and analytic disturbances (lymphocytopenia of $790 \mathrm{cells} / \mathrm{mcl}$, D-dimer 0'55 $\mathrm{gg} / \mathrm{ml}$, C-reactive protein 10'2 mg/dl, lactate 
dehydrogenase $338 \mathrm{U} / \mathrm{L}$ ). He was admitted to the IMS on hydroxychloroquine, lopinavir/ritonavir and LMWH 80 mg b.i.d.

He progressed into acute respiratory distress syndrome with radiological and analytical deterioration (lymphocytopenia of $410 \mathrm{cells} / \mathrm{mcl}$, D-dimer $21^{\prime} 4 \mu \mathrm{g} / \mathrm{ml}$, lactate dehydrogenase $820 \mathrm{U} / \mathrm{L}$ ). High doses of methylprednisolone and two doses of tocilizumab were administered and he was admitted to ICU for MV on his 18th day of symptoms. During the following days and because of persistently high D-dimer levels, he was performed a CT pulmonary angiogram that showed a bilateral pulmonary embolism. He was discharged back to the IMS on his 31st day of symptoms, remaining respiratory stable and with improvement of the analytical alterations.

When waking up in the ICU he was conscious without focal neurological deficits but with remarkable global altered mental status. He was confused with inattention, global disorientation and an inconsistent speech with delusional ideas and sensory-perceptual phenomena, with fluctuating symptoms and psychomotor agitation. He was seen by the Psychiatrist and treated with risperidone until full recovery one week later.

Systemic, infectious and other toxic-metabolic conditions were ruled out. An electroencephalogram showed moderate encephalopathy and a brain MRI revealed on DWI sequence acute bilateral ischemic lesions affecting both frontal and left occipital lobes, with several microhemorrhagic foci on SWI sequence (Fig. 1 d, e, f). Cardioembolic source and acquired antiphospholipid syndrome were ruled out. COVID-19-associated coagulopathy was suspected as the possible main etiology of the strokes, supported by the presence of pulmonary embolism as another thrombotic complication in this patient.

\section{Discussion}

To our knowledge acute confusional state and encephalopathy have been described in COVID-19 patients, even as the initial presentation of the disease, related to the systemic condition without a primary neurological cause [4]. We present two cases of COVID-19 patients between 60-65 years old without previous neurological conditions and acutely altered mental status as the main manifestation of multiple cerebral infarcts after a long stay in ICU.

A high frequency of cerebrovascular events is being reported in COVID-19 patients, potentially affecting multiple vascular territories, and probably associated with a sepsis induced hypercoagulable state. Although the most common findings in stroke are focal neurological deficits, ischemic lesions related to this condition might be due to multifocal and distal vessel occlusions which could more frequently manifest as an altered mental status rather than as a focal neurological deficit [5]. Furthermore, this could be especially relevant in younger patients without previous comorbidities, who are not so prone to present altered mental status during an acute disease and in whom secondary causes of this condition must be taken into account. 
In view of the above and supported by our evidence, we consider important to rule out cerebrovascular entities by performing periodic neurological examinations preceded by transient withdrawal of sedatives in those intubated patients with COVID-19 and suspicion of COVID-19-associated coagulopathy. We suggest to perform a brain MRI in those patients with acutely altered mental status on examination and in those who cannot be clinically evaluated [6].

Full-dose anticoagulation would probably be indicated and could prevent complications in these patients, being necessary more studies to confirm this supposition [7].

\section{Conclusions}

In conclusion, acutely altered mental status might be the main manifestation of multiple brain infarctions in critically ill COVID-19 patients and it should be specially considered in those with suspected COVID-19associated coagulopathy. Full-dose anticoagulation and clinical-radiological monitoring might reduce neurological consequences in those patients.

\section{Declarations}

Verbal informed consent was obtained from the patient's relatives to the submission of the case report.

\section{References}

1. Harapan H, Itoh N, Yufika A. Coronavirus disease 2019 (COVID-19): A literature review.J Infect Public Health. 2020 Apr 8. pii: S1876-0341(20)30432-9. doi: 10.1016/j.jiph.2020.03.019.

2. Mao L, Jin H, Wang M et al. Neurologic Manifestations of Hospitalized Patients With Coronavirus Disease 2019 in Wuhan, China. JAMA Neurol. 2020 Apr 10. doi: 10.1001/jamaneurol.2020.1127.

3. Spiezia L, Boscolo A, Poletto F et al. COVID-19-Related Severe Hypercoagulability in Patients Admitted to Intensive Care Unit for Acute Respiratory Failure. Thromb Haemost. 2020 Apr 21. doi: 10.1055/s-0040-1710018.

4. Filatov A, Sharma P, Hindi F, Espinosa PS. Neurological Complications of Coronavirus Disease (COVID-19): Encephalopathy. Cureus. 2020 Mar 21;12(3): e735. doi: 10.7759/cureus.7352.

5. Ferro JM, Caeiro L, Verdelho A. Delirium in acute stroke. Curr Opin Neurol. 2002 Feb;15(1):51-55. doi: 10.1097/00019052-200202000-00009.

6. Helms J, Kremer S, Merdji H et al. Neurologic features in severe SARS-CoV-2 infection. N Engl J Med. 2020 Apr 15. doi: 10.1056/NEJMc2008597.

7. Tang N, Bai H, Chen X, Gong J, Li D, Sun Z. Anticoagulant treatment is associated with decreased mortality in severe coronavirus disease 2019 patients with coagulopathy. J Thromb Haemost. 2020 May;18(5):1094-1099. doi: 10.1111/jth.14817.

\section{Figures}




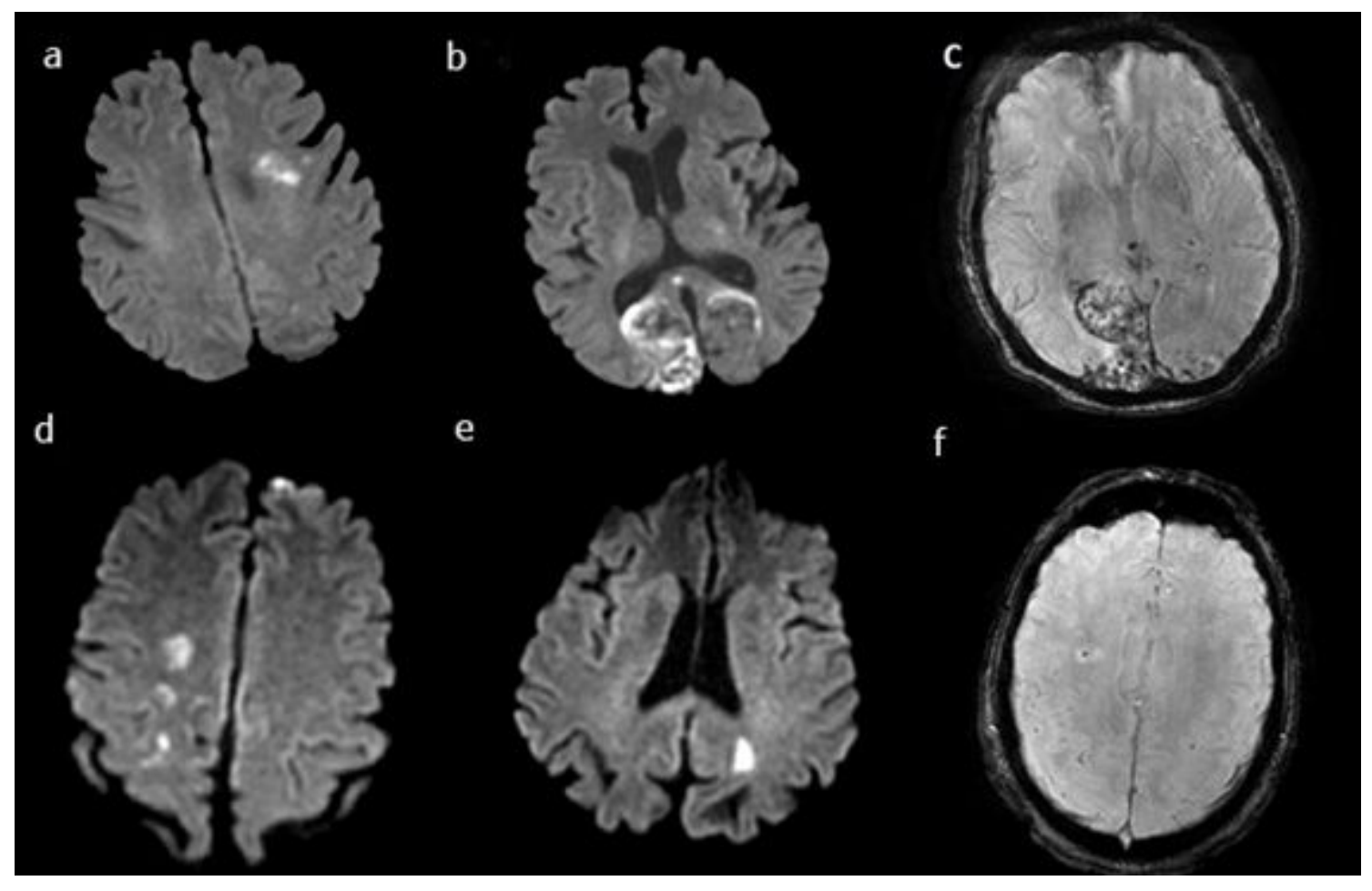

Figure 1

a, b) Case 1. Multiple ischemic lesions visible on DWI sequences (brain MRI) affecting the left frontal and temporal lobes and both occipital lobes. c) Case 1. Extensive areas of hemorrhagic transformation on SWI sequences (brain MRI) affecting both occipital lobes. d, e) Case 2. Acute bilateral ischemic lesions affecting both frontal and left occipital lobes on DWI sequences. f) Case 2. Several microhemorrhagic foci affecting both frontal and left occipital lobes on SWI sequences. 